\title{
The flat polyp: does too much flattening occur with full air insufflation on colonoscopy?
}

Flat polyps have been described by our Japanese colleagues. Recently flat polyps have also been recognized in the West and have been noticed to have a higher incidence of significant neoplastic histology [1]. We describe two cases of a flat polyp missed or almost missed at colonoscopy, perhaps due to the effects of full insufflation.

A 53-year-old healthy woman participated in a PillCam Colon 2 study in which PillCam Colon 2 capsule endoscopy (PillCam Colon 2; Given Imaging, Yoqneam, Israel) was compared with optical colonoscopy [2]. The indication for colonoscopy was painless rectal bleeding. The PillCam Colon 2 capsule study was performed in the morning, and after the capsule was passed in the afternoon a colonoscopy was performed. The patient received $2.5 \mathrm{mg}$ midazolam and $50 \mathrm{mg}$ pethidine intravenously. A Fujinon $250 \mathrm{~W}$ colonoscope (Fujinon Corp., Saitama, Japan) was advanced to the ileum. The distal ileum and cecum were carefully examined, and the appendiceal orifice and ileocecal valve were identified. Withdrawal time was 25 minutes. Internal hemorrhoids were present. No lesions were noticed in the cecum.

When the PillCam Colon 2 study was reviewed we were notified that a $15-\mathrm{mm}$ sessile polyp was seen in the cecum ( Fig. 1). The patient was invited to a repeat colonoscopy for verification of this finding and removal of the polyp. The second colonoscopy confirmed the presence of a completely flat lesion $2 \mathrm{~cm}$ in size ( Fig. 2). The histology examination identified a tubulovillous adenoma.

In the second case, a 69-year-old woman underwent colonoscopy with an Olympus narrow-band imaging colonoscope (PCF180; Olympus Optical Co., Ltd., Tokyo, Japan) for surveillance after colonoscopic polypectomy, and a flat lesion was found in the ascending colon ( $\mathbf{F i g}$. 3) which was not removed during this colonoscopy because the colon was full of residual stool which could not be washed and sucked out. The patient was invited for repeat colonoscopy which, on full insufflation, could not at first detect the polyp ( $\bullet$ Fig. 4). Since the location of the polyp in relation to the ileocecal valve was well
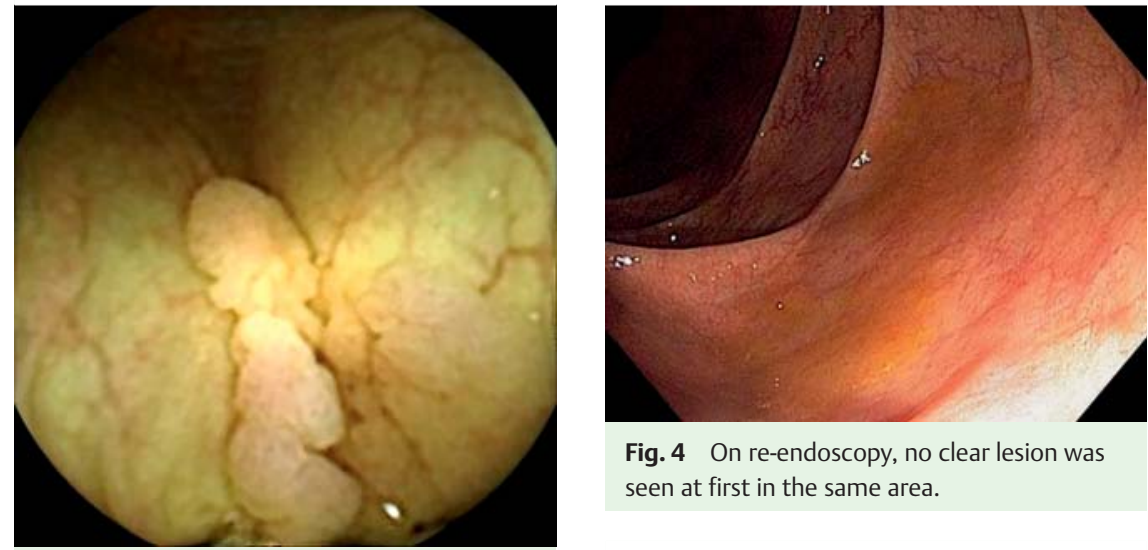

Fig. 4 On re-endoscopy, no clear lesion was seen at first in the same area.

Fig. 1 A 15-mm elevated polyp in the cecum visualized by PillCam Colon 2 .

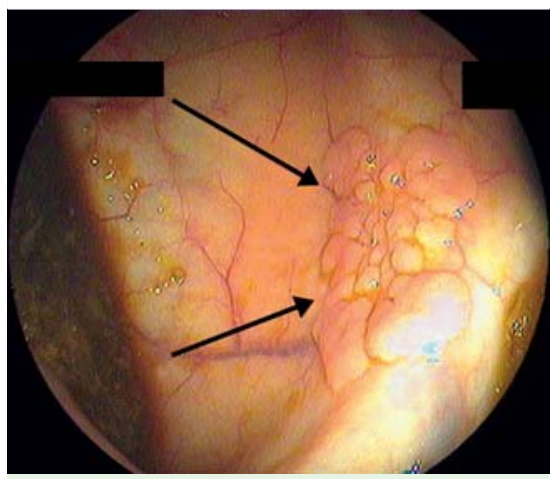

Fig. 2 Same cecal lesion as in $\bullet$ Fig. 1, but the polyp is completely flat.

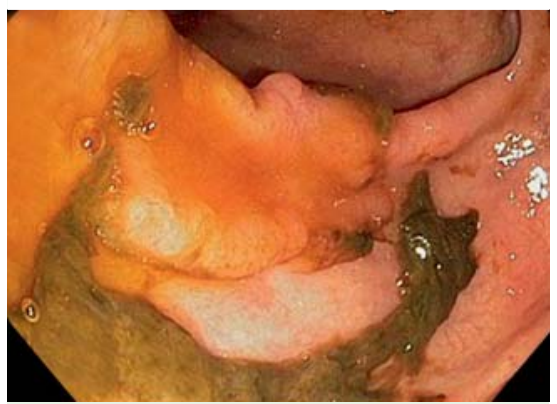

Fig. 3 Flat adenoma in the ascending colon with insufficient bowel cleaning.

known from previous colonoscopy images, the search was continued for several minutes and acetic acid used, which finally revealed a rather large area of flat polyp, about $3 \mathrm{~cm}$ in size ( $\mathrm{Fig}$. 5). The lesion was finally removed piecemeal ( $\bullet$ Fig. 6) and proved on histology to be a sessile serrated adenoma.

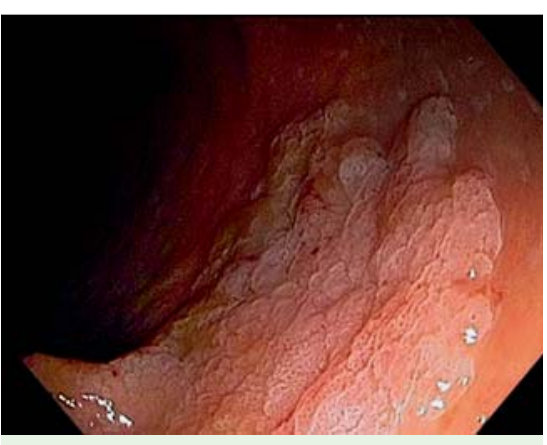

Fig. 5 A flat lesion with villous structure was clearly seen only after spraying with acetic acid.

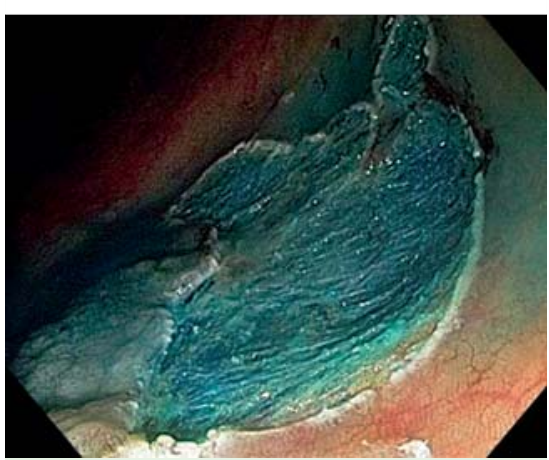

Fig. 6 Ascending colon after saline-assisted piecemeal polypectomy.

These cases show that when the colon is fully distended, flat lesions may be overlooked. The polyp in the first case, located in the nondistended cecum, was readily recognized at PillCam Colon 2 colonoscopy as a $1.5-\mathrm{cm}$ sessile protruding finding. Yet this same polyp at colonoscopy in a distended cecum appeared as a completely flat lesion. The discrepancy was even more pronounced in the second case. It appears that insufflation of the colon at 
colonoscopy distended the cecal wall, thus stretching this sessile tubulovillous adenoma and turning it into a flat lesion that was difficult to recognize. In the European colon capsule study, five sessile polyps were diagnosed at colonoscopy which did not have a flat appearance at capsule endoscopy (personal communication, Jacques Devière) [3].

Some "flat" lesions we encounter may be flat only due to stretching of the colonic wall at colonoscopy. It may be wise to look for these lesions on the way in to the cecum before the colonic walls are distended with air, or to examine the segments of the colon after air has been suctioned from the colon. This may make recognition of "man-made flat lesions" easier. It remains to be determined whether capsule colonoscopy has an advantage over conventional colonoscopy for the detection of flat lesions.

Competing interests: None

Endoscopy_UCTN_Code_CCL_1AD_2AB

\section{S. N. Adler ${ }^{1}$, Y. C. Metzger ${ }^{2}$, T. Roesch}

1 Division of Gastroenterology,

Bikur Cholim Hospital, Jerusalem, Israel

2 School of Medicine, The Hebrew University, Jerusalem, Israel

3 Charité University Hospitals, Berlin, Germany

\section{References}

1 Soetikno RM, Kaltenbach T, Rouse RV et al. Prevalence of nonpolypoid (flat and depressed) colorectal neoplasms in asymptomatic and symptomatic adults. JAMA 2008; 299: $1027-1035$

2 Eliakim R, Yassin K, Niv Y et al. Prospective multicenter performance evaluation of the second-generation colon capsule compared with colonoscopy. Endoscopy 2009; 41: $1026-1031$

3 Van Gossum A, Navas MM, Fernandez-Urien I et al. Capsule endoscopy versus colonoscopy for the detection of polyps and cancer. $\mathrm{N}$ Engl J Med 2009; 361: 264-270
Bibliography

DOI 10.1055/s-0029-1244164

Endoscopy 2010; 42: E167-E168

(c) Georg Thieme Verlag KG Stuttgart · New York . ISSN 0013-726X

Corresponding author

\section{S. N. Adler, MD}

Gastroenterology

Bikur Cholim Hospital

5 Strauss Street

POB 492

Jerusalem 91004

Israel

Fax: +972-2-6464378

nasnadler@gmail.com 